\title{
ROM JENSEITS DER GRENZE: KLIENTELKÖNIGREICHE UND DER IMPACT OF EMPIRE
}

\author{
GÜNTHER SCHÖRNER
}

\section{EINLEITUNG}

Es ist eine allgemein bekannte Tatsache, dass die römische Aktionssphäre nicht mit dem Gebiet, das von römischen Institutionen verwaltet wurde, identisch ist. Wie Whittaker überzeugend nachweisen konnte, unterteilten die Römer in der frühen Kaiserzeit die Welt in drei unterschiedliche Zonen: ${ }^{1}$ das unter direkter römische Verwaltung stehende Territorium, das nicht unter direkter römischer Verwaltung stehende Territorium, und die äußere Peripherie. Entscheidend dabei ist auch, dass nicht der Raum das grundlegende Konzept ist, sondern die Verfügungsgewalt, das imperium-ein Begriff, der erst sekundär auch eine raumhafte Bedeutung annimmt. ${ }^{2}$ Legt man eine lokal-geographische Gliederung zugrunde, so ist das Gebiet der Oecumene in verschiedene provinciae usque ad oceanum eingeteilt, die aber nicht notwendigerweise identisch sind mit den Provinzen als Verwaltungseinheiten; so gibt es PseudoProvinzen wie Sarmatia oder Germania, die für Gebiete stehen, für die die Römer Kontrolle in Anspruch nehmen, nicht aber Verwaltung. ${ }^{3}$

${ }^{1}$ Mein Dank gilt Prof. Dr. O. Hekster (Nijmegen) und Dr. T. Kaizer (Durham) für die Einladung zum Neunten Workshop des Internationalen Netzwerkes ,Impact of Empire. Für wichtige Auskünfte und Kommentare danke ich R. Hingley (Durham), T. Kaizer (Durham), T. Kleinschmidt (Jena), A. Levin (Florenz/Potenza) und meiner Frau H. Schörner.

C.R. Whittaker, Frontiers of the Roman Empire (Baltimore 1994), 12-18.

2 J.S. Richardson, ,Imperium Romanum: Empire and language of power, Journal of Roman studies 81 (1991), 1-9.

${ }^{3}$ Zur römischen Sichtweise von Raum und Grenzen: C.R. Whittaker, ,Mental maps and frontiers. Seeing like a Roman', in: P. McKechnie (Hrsg.), Thinking like a lawyer. Essays on legal history and general history for John Crook on his eightieth birthday (Leiden 2002), 81-112; Wiederabdruck in: C.R. Whittaker, Rome and its frontiers (LondonNew York 2004), 63-87; vgl. auch S.P. Mattern, Rome and the enemy. Imperial strategy in the principate (Berkeley-Los Angeles-London 1999), 24-80; wichtig ist, dass römische Gesetze sowohl für die Provinzen als auch für die Klientelreiche galten: D. Braund, Rome and the friendly king. The character of client kingship (New York 1984), 66. 
Überträgt man diese Konzeption auf die Klientelstaaten, so gehören sie notwendigerweise zu diesen provinciae. ${ }^{4}$ Teil des Imperiums zu sein bedeutete also, unter imperialer Kontrolle zu stehen, nicht unbedingt aber eine Provinz unter direkter römischer Administration mit Statthalter zu sein. Diese Diskrepanz zwischen verwaltetem und kontrolliertem Gebiet war natürlich grundlegend für die römische Wahrnehmung von Klientelkönigen und deren Reiche. Es mindert also nicht den Herrschaftsanspruch Roms, wenn Gebiete am Rande des direkt verwalteten Territoriums von Königen beherrscht wurden, die in enger Beziehung zu Rom standen. ${ }^{5}$ Der enge, direkte Konnex zwischen Rom als Machtzentrum und diesen so genannten Klientelkönigen wird in der lateinischen Terminologie evident, wie sie literarische Quellen belegen: Sueton schreibt, dass Augustus die einheimischen Regenten als membra partesque imperii betrachtete. ${ }^{6}$ Die Klientelkönige wurden offiziell mehrfach als socii et amici populi Romani bezeichnet. ${ }^{7}$ Trotz dieser Terminologie lag die oberste Verfügungsgewalt nach römischem Verständnis in

${ }^{4}$ Grundlegende Literatur zu Klientelkönigen aus althistorischer Sicht: P.C. Sands, The client princes of the Roman Empire under the Republic (Cambridge 1908); M.R. Cimma, Reges socii et amici populi Romani (Mailand 1976); Braund 1984, a.a. O. (Anm. 2); D. Braund, ,Client kings', in: D. Braund (Hrsg.), The administration of the Roman empire (241 BC-AD 193) (Exeter 1988), 69-96; R.D. Sullivan, Near Eastern royalty and Rome, 10030 BC (Toronto 1990); E. Paltiel, Vassals and rebels in the Roman Empire. Julio-Claudian policies in Judaee and the kingdoms of the East (Brüssel 1991); M. Sommer, Roms orientalische Steppengrenze. Palmyra-Edessa-Dura Europos-Hatra. Eine Kulturgeschichte von Pompeius bis Diocletian (Stuttgart 2005), 58-66; und jetzt T. Kaizer and M. Facella (eds.), Kingdoms and Principalities in the Roman Near East (Stuttgart 2010). Zu Klientelkönigreichen im Norden und Westen: E. Will, ,Römische ,Klientel-Randstaaten' am Rhein? Eine Bestandsaufnahme', Bonner Jahrbücher 187 (1987), 1-62; L.F. Pitts, ,Relations between Rome and the German ,kings' on the Middle Danube in the first to fourth centuries AD', Journal of Roman Studies 79 (1989), 1945-1958; J. Creighton, Coins and power in Late Iron Age Britain (Cambridge 2000), 55-79; J. Creighton, Britannia. The Creation of a Roman province (London-New York 2006), passim, vor allem 14-34; D. Mattingly, An Imperial Possession: Britain in the Roman Empire, 54 BC-AD 409 (London 2006), 68-84.

${ }^{5}$ Vergleichbar ist die Wichtigkeit von Gesandtschaften fremder Könige nach Rom, die nach römischen Verständnis die Machtverhältnisse zum Ausdruck bringen; zentrale Belege: Res Gestae Divi Augusti 31-32; Sueton, Augustus 21.3; Horaz, Carmen saeculare 55 f.; Tacitus, Annales 4.26; Strabo 15.1.73. grundlegend: J. Gagé, ,Lempereur et les rois: Politique et protocol', Revue historique 221 (1959), 221-260; Mattern 1999, a. a. O. (Anm. 3), 172 f.; zu Geiseln s. u. Anm. 68.

${ }^{6}$ Sueton, Augustus 48.

7 Grundlegend: W. Dahlheim, Struktur und Entwicklung des römischen Völkerrechts im dritten und zweiten Jahrhundert v. Chr. (München 1968); vgl. A. Coșkun, ,Freundschaft und Klientelbindung in Roms auswärtigen Beziehungen, in: A. Coşkun (Hrsg.), Roms auswärtige Freunde in der späten Republik und im frühen Prinzipat (Göttingen 2005), $1-30$. 
Rom, das heißt beim Volk und Senat von Rom bzw. beim Kaiser selbst. Tacitus benannte dementsprechend die Herrschaft eines Klientelkönigs als donum populi Romani. ${ }^{8}$ Ein Klientelreich kann deshalb in eine Provinz unter Verwaltung eines römischen Statthalters umgewandelt werden (und wieder zurück). ${ }^{9}$ Für die von Klientelkönigen beherrschten Gebiete trifft somit der von Richard Duncan-Jones geprägte Begriff der ,occupation without annexation ebenfalls zu. ${ }^{10}$

Während das römische Vorgehen gegenüber den reges im Westen und Osten gut untersucht ist, besteht jedoch noch großer Forschungsbedarf hinsichtlich der Auswirkungen, die die Verbindungen zu Rom in den Herrschaftsgebieten der Klientelkönige selbst hatten, wobei grundsätzlich vor allem zu fragen ist, wie weit die Einflüsse reichen, das heißt, welche gesellschaftlichen Gruppen in diesen Grenzregionen überhaupt betroffen sind. Diese Fragestellung hat vor allem einen Vorteil: Sie legt den Schwerpunkt der Analyse auf die Peripherie und nicht auf Rom als Zentrum, das nach einer, Grand Strategy' vorgehend die alleinige Handlungsmacht besitzt. ${ }^{11}$ Im Folgenden sollen die Modi der Adaption römischer Kulturelemente in zwei verschiedenen Bereichen überprüft werden, im Bereich der Kulte und im Bereich der materiellen Kultur.

\section{II. ÜBERNAHME UND ADAPTION RÖMISCHER Kulturelemente in KLIENTELREICHEN}

\section{Rituale}

Eine der wichtigsten Charakteristika, die für die Konstitution der Beziehung zwischen Rom und den Klientelreichen entscheidend sind, ist die

8 Tacitus, Annales IV 5, 3: Mauros Iuba rex acceperat donum populi Romani; vgl. auch Tacitus, Annales 12.45.5 über die Einsetzung des armenischen Königs.

9 Whittaker 1994, a. a. O. (Anm. 1), 54; vgl. auch das Schema bei Creighton 2006, a. a. O. (Anm. 4), 18 Abb. 1.1; signifikant auch der Begriff des rex datus: Res Gestae Divi Augusti 33; Gagé 1959, a. a. O (Anm. 5), 252-255; als Motiv der Münzprägung: E.W. Swoboda, ,Rex Quadis datus', Carnuntum Jahrbuch 2 (1956), 5-12; R. Göbl, ,rex ... datus: Ein Kapitel von der Interpretation numismatischer Zeugnisse und ihren Grundlagen', Rheinisches Museum 104 (1961), 70-80; M. Rosenbaum-Alföldi, ,Nochmals, Rex Quadis datus", Numismatische Zeitschrift 106-107 (1999), 91-94.

${ }^{10}$ R. Duncan-Jones, ,Ti. Claudius Subatianus Aquila: „first prefect of Mesopotamia“", Zeitschrift für Papyrologie und Epigraphik 36 (1979), 255-262.

${ }^{11}$ E.N. Luttvak, The Grand Strategy of the Roman Empire, (Baltimore 1979); hierzu unter anderem: Mattern 1999, a. a. O. (Anm. 3), 81-122; Whittaker 2004, a.a. O. (Anm. 3), 28-49. 
Bindung an Rom durch Kulte. Der dabei gebräuchlichste Weg ist die Etablierung eines Kultes für den römischen Kaiser, wie anhand epigraphischer, literarischer und archäologischer Evidenz bezeugt ist. ${ }^{12}$ Herodes der Große ließ Tempel für Augustus errichten, Kulte für Augustus wurden in den herodianischen Hauptstädten Caesarea maritima, Samaria-Sebaste und Caesarea Philippi-Paneas etabliert. ${ }^{13}$ Einzige Ausnahme scheint Tiberias gewesen zu sein, für das bisher noch keine entsprechende Evidenz nachgewiesen ist. ${ }^{14}$ Der Kaiserkult wurde als so bestimmend angesehen, dass sogar eine Verbindung zur jüdischen Religion geschaffen wurde: Während römische Herrscher Opfer an Yahweh im Tempel von Jerusalem darbrachten, so opferten die Hohen Priester zum Wohle der Kaiser. ${ }^{15}$

Die weite Verbreitung des Kaiserkultes in den provinciae außerhalb des römischen Reiches ist durchaus gebräuchlich wie das templum Augusti von Muziris in Indien auf der Tabula Peutingeriana beweist. ${ }^{16}$

Noch signifikanter als die Verehrung des römischen Kaisers, die durchaus nach den eigenen kultischen Vorstellungen konzipiert und

12 Allgemein: M. Clauss, Kaiser und Gott. Herrscherkult im römischen Reich (München 2001) (mit reicher Lit.); H. Cancik-K. Hitzl (Hrsg.), Die Praxis der Herrscherverehrung in Rom und seinen Provinzen (Tübingen 2003); G. Woolf, ,Divinity and power in ancient Rome, in: N. Brisch (Hrsg.), Religion and Power: Divine Kingship in the Ancient World and Beyond (Chicago 2008), 243-260; zum Kaiserkult in Klientelreichen: Braund 1984, a. a. O. (Anm. 3), 112-114; Mauretanien: D. Roller, The World of Iuba II and Kleopatra Selene: Royal Scholarship at Rome\%s African Frontier (London-New York 2003), 126 f.; Palästina: M. Bernett, Der Kaiserkult in Judäa unter den Herodiern und Römern: Untersuchungen zur politischen und religiösen Geschichte Judäas von 30 v. bis 66 n. Chr. (mit der älteren Lit.) (Tübingen 2007).

13 Allgemein: S. Japp, Die Baupolitik Herodes' des Großen: die Bedeutung der Architektur für die Herrschaftslegitimation eines römischen Klientelkönigs (Rahden 2000), 56-60; zur baulichen Gestalt der Kaiserkulttempel zusammenfassend zuletzt: S. Japp, ,Tradition und Innovation im Bauprogramm Herodes' des Großen am Beispiel der Heiligtümer, in: K.S. Freyberger-A. Henning-H. von Hesberg (Hrsg.), Kulturkonflikte im Vorderen Orient an der Wende vom Hellenismus zur römischen Kaiserzeit (Rahden 2003), 291-303; Caesarea: H. Hänlein-Schäfer, Veneratio Augusti: eine Studie zu den Tempeln des ersten römischen Kaisers (Rom 1985), 201-203 Kat. A 48; Sebaste: ebenda 199-201 Kat. A 47; Panias: ebenda 198 f. Kat. A 46; Z.U. Ma'Oz, Baniyas, the Roman Temples (Qazrin 2009), 50-60.

${ }^{14}$ S. Freyne, ,The Galilean world of Jesus, in: P.F. Ester (Hrsg.), The early Christian world I (London-New York 200o), $127 \mathrm{f}$.; R. Yat Tin Lee, Romanization in Palestine. A study of urban development from Herod the Great to AD 70 (Oxford 2003), 78.

15 Yat Tin Lee 2003, a.a. O. (Anm. 14), 78.

${ }^{16}$ Hänlein-Schäfer 1985, a.a. O. (Anm. 13), 254 Kat. A 66; zum antiken Muziris: K.P. Shajan-R. Tomber-V. Selvakumar-P.J. Cherian, ,Locating the ancient port of Muziris: fresh findings from Pattanam, Journal of Roman Archaeology 17 (2004), 312320. 
durchgeführt werden konnte, ist jedoch die Übernahme von römischen Ritualen als einem fremden System komplexer Aktionen.

So zeigen Münzen, die von Königen in Süd- und Ostengland vor der Eroberung der Insel durch Claudius geprägt wurden, die Darstellung von Ritualen, die gemäß Kultvorstellungen Roms durchgeführt wurden. Die Übernahme ritueller Performanz verdeutlicht am besten eine Münze von Cunobelin, auf deren Rückseite eine Person zwischen zwei Dreifüßen zu sehen ist. ${ }^{17}$ Der foculus war eines der typischsten Kultgeräte beim römischen Opfer, da auf ihm die libatio ture ac vino, das Opfer von Weihrauch und Wein, als praefatio vor dem blutigen Opfer oder eigenständiges Ritual durchgeführt wurde. ${ }^{18}$ Die Darstellung des Dreifußes auf der Münze-verstärkt durch eine Verdoppelung-weist deshalb in verkürzter, aber dafür besonders konzentrierter Form auf die Übernahme des römischen Opferrituals durch einen englischen Klientelkönig im Britannien vor der Eingliederung als Provinz in das Imperium Romanum hin. ${ }^{19}$ Andere römische Ritualgeräte sind auf einem Münztyp, der von Dubnovellaunus, Tasciovanus und Cunobelin geprägt wurde, wiedergegeben, auf dessen Rückseite ein auf einem Thron sitzender Mann dargestellt ist, der einen lituus hält. ${ }^{20}$ Zwar ist der lituus auch Zeichen des imperium und somit der Befehlsgewalt, doch ist die Verbindung mit dem Ritual der Auspizien grundlegend, so dass zumindest eine typisch römische Kultinsignie Eingang in die herrschaftliche Ikonographie einheimischer Herrscher gefunden hat. ${ }^{21}$

Wie wichtig die Übernahme römischer Rituale ist, wird evident am Suovetaurilienrelief des Bogens von Susa, dem antiken Segusio, das Donnus, der als König über dieses Gebiet in den Westalpen herrschte, zu seiner Hauptstadt gemacht hatte. ${ }^{22}$ Donnus' Sohn Cottius schloss mit

17 Creighton 2000, a.a. O. (Anm. 4), 205 Abb. 7.8.

18 Grundlegende Bearbeitung der foculi: U. Klatt, ,Römische Klapptische. Drei- und vierbeinige Stützgestelle aus Bronze und Silber', Kölner Jahrbuch 28 (1995), 349-573, vor allem 383-419; zum Gebrauch beim Opfer: ebenda 416f. 430-438; vgl. auch A.V. Siebert, Instrumenta sacra. Untersuchungen zu römischen Opfer-, Kult- und Priestergeräten (Berlin 1999), 93-98; Zur praefatio: J. Scheid, Romulus et ses frères. Le collège des frères arvales, modèle du culte public dans la Rome des Empereurs (Rom 1990), 326-333.

19 Allgemein: Creighton 2000. a. a. O. (Anm. 4), 201-202 (mit weiteren Belegen).

20 Creighton 2000, a. a. O. (Anm. 4), 210-213 Abb. 7.8.

21 Siebert 1999, a. a. O. (Anm. 18), 130-132 (mit der älteren Lit.).

22 Zur Geschichte des Gebiets: J. Prieur, La province romaine des Alpes Cottiennes (Lyon 1968); J. Prieur, ,L'histoire des régions alpestres (Alpes Maritimes, Cottiennes, Graies et Pennines) sous le haut-empire romain (Ier-IIIer siècles après J.-C.)', in: Aufstieg und Niedergang der Römischen Welt II 5,2 (Berlin 1976), 630-656; G. Walser, Studien 
Augustus einen Vertrag, so dass er zum praefectus civitatium wurde. Er veranlasste im Jahr $9 / 8$ v. Chr. die Errichtung eines Bogens als Ausdruck seiner Eintracht mit dem römischen Kaiser. ${ }^{23}$ Thema des Bildschmuckes ist ein Opfer, dass Cottius gemeinsam mit Augustus durchführte: ${ }^{24}$ Cottius in Toga wird von Liktoren begleitet und entspricht dabei ganz der Darstellung des Augustus auf der anderen Seite des Bogens. Sowohl Cottius als auch Augustus begehen eine kultische Reinigung mit Stier, Schaf und Schwein als Opfertieren (Abb. 1). Das Lustrationsopfer wird also nach römischen Vorstellungen durchgeführt, die Performanz entspricht einer in Rom durchgeführten suovetaurilia. ${ }^{25}$ Auch wenn Marcus Iulius Cottius nicht mehr nominell König war, so entspricht seine Platzierung in der Friesmitte ganz der des Augustus und ist Ausdruck seines Selbstverständnisses. Das Relief am Bogen von Susa ist somit ein Beispiel, wie lokale Potentaten römische Rituale nutzen, um ihr Verhältnis sowohl nach außen zum römischen Kaiser als auch nach innen gegenüber den eigenen Bürgern zum Ausdruck zu bringen. ${ }^{26}$ Cottius scheint insbesondere seine Stellung gegenüber Rom erfolgreich behauptet zu haben: Sein Sohn Cottius II. wurde wieder zum König ernannt. ${ }^{27}$ Grundsätzlich besteht bei Überbetonung der kultischen Verbindungen zum Zentrum

zur Alpengeschichte in antiker Zeit (Stuttgart 1994); G. Barruol, Les peuples préromaines du sud-est de la Gaule (2. Auflage; Paris 1999); T. Bechert, Die Provinzen des Römischen Reiches. Einführung und Überblick (Mainz 1999), 187-190.

${ }^{23}$ E. Ferrero, L'arc d'Auguste à Susa (Paris 1901); F. Studniczka, Über den Augustusbogen in Susa', Jahrbuch des Deutschen Archäologischen Instituts 18 (1903), 1-24; J. Prieur, ,Les arcs monumentaux dans les Alpes occidentales: Aoste, Suse, Ais-les-Bains', in: Aufstieg und Niedergang der Römischen Welt II 12, 1 (Berlin 1985), 442-475; S. De Maria, Gli archi onorari di Roma e dell'Italia romana (Rom 1988), 329 f.; D. Fogliato, L'arco di Augusto a Susa (Collegno 1992); P. Pensabene, ,Monumenti augustei delle province alpine occidentali: cultura, architettonica, materiali e commitenza, in: M. Sapelli Ragni (Hrsg.), Studi di archeologia in memoria di Liliana Mercando (Turin 2005), 211-229.

${ }^{24}$ S. Reinach, Répertoire des reliefs grecs et romains I (Paris 1909), 418-421; B.M. Felleti Maj, ,Il fregio commemorativo dell'arco di Susa', Rendiconti della Pontificia Accademia 33 (1960/61), 129-153; M.C. Calvi, ,Osservazioni sul fregio dell'arco di Susa', Archeologia Classica 28 (1976), 115-125; S. De Maria, ,Apparato figurativo nellarco onorario di Susa, Rivista d'Archeologia 1 (1977), 44-52; K. Moede, ,Der Augustusbogen von Susa. Römische Rituale außerhalb Roms, in: F. und T. Hölscher, Römische Bilderwelten. Von der Wirklichkeit zum Bild und zurück (Heidelberg 2007), 133-144.

${ }^{25}$ RE XIII 2 (1927), 2029-2039 s.-v. lustratio (F. Boehm); U.W. Scholz, ,Suovetaurilia und Solitaurilia, Philologus 117 (1973), 3-28; F. Fless, Thesaurus Cultus et Rituum Antiquorum I (Zürich 2004), 33-58 s. v. ,Prozession, römisch'; D. Baudy, Römische Umgangsriten (Berlin 1998), 106-114; F. Stilp, Mariage et suovetaurilia. Etude sur le soi-disant, Autel de Domitius Ahenobarbus', 26. Supplement Rivista d'Archeologia (2001).

${ }^{26}$ Moede 2007, a. a. O. (Anm. 24), 140-142.

27 Braund 1984, a.a. O. (Anm. 3), 84. 


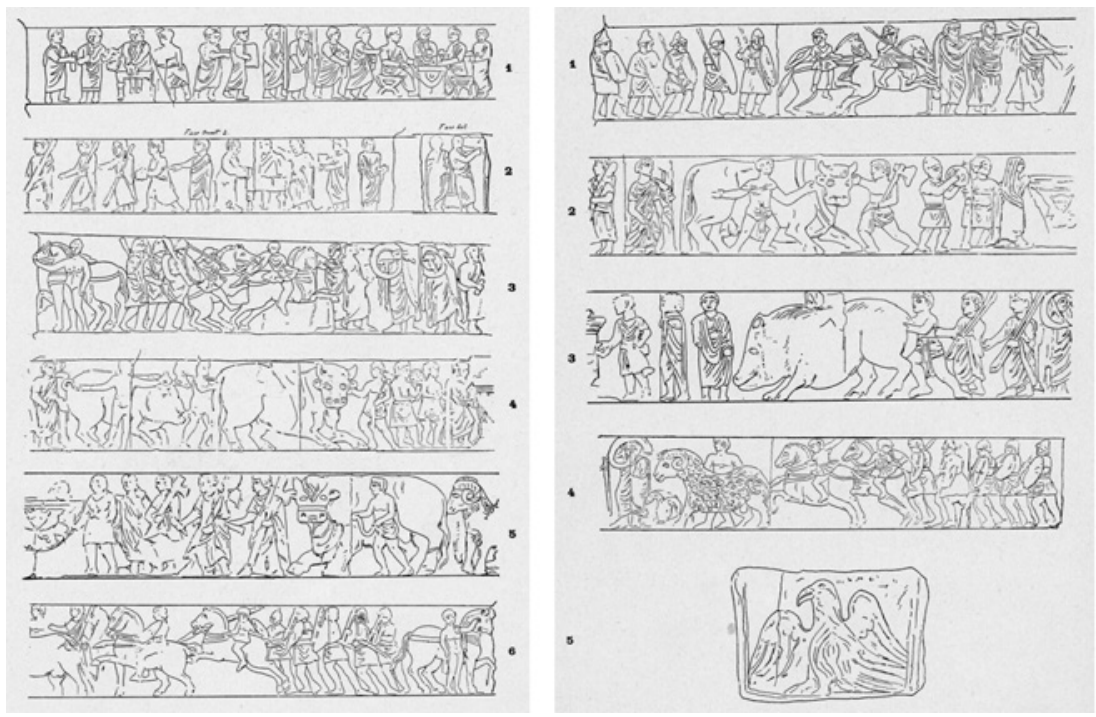

Abb. 1: Relieffries des Bogens von Susa:

Augustus (a) und Cottius (b) beim Opfer

freilich die Gefahr, dass enge kultische Beziehungen zwischen König und einheimischen Untertanen aufgegeben werden und sowohl sich beide Seiten entfremden als auch eine Form von ,kolonialer Religion' etabliert wird. $^{28}$

\section{Materielle Kultur}

\section{a. Bautechnik}

Die materielle Kultur spiegelt besonders eindrucksvoll Umfang und Nachhaltigkeit des ,Impact of Empire in den Klientelkönigreichen wider.

Ein besonders wichtiger gemeinsamer Faktor der regna ist die Adaption römischer Bautechniken. Am deutlichsten wird dies an der Ver-

${ }^{28} \mathrm{Zu}$ römischen Ritualen außerhalb Roms: C. Ando, ,Exporting Roman religion, in: J. Rüpke (Hrsg.), A companion to Roman religion (Malden-London 2007), 429445; zu kolonialer Religion als ideologische Komponente von Weltreichen grundlegend: C.M. Sinopoli, ,The archaeology of empires', Annual Review of Anthropology 23 (1994), 159-180, vor allem 167f.; Beispiele aus nicht-römischer Perspektive: G.W. ConradA.A. Demarest, Religion and empire: The dynamics of Aztec and Inca expansionism (Cambridge 1984); E.M. Brumfiel, ,Aztec hearts and minds: religion and the state in the Aztec empire, in: S. Alcock-T. D'Altroy-K. Morrison-C. Sinopoli (Hrsg.), Empires. Perspectives from archaeology and history (Cambridge 2001), 283-310. 
breitung von opus reticulatum und opus caementicium..$^{29}$ Die Verwendung von Retikulatmauerwerk ist für herodianische Zeit gut in Iudaea bezeugt, so am Dritten Winterpalast in Jericho, verschiedenen Gebäuden in Paneas, in Masada, in Jerusalem und in Caesarea maritima. ${ }^{30}$ Sogar kampanische Pozzolan-Erde wurde nach Caesarea importiert, um den Hafen Sebastos zu errichten, ${ }^{31}$ weil geeignete Rohstoffe für die adäquate Anwendung der opus caementicium-Technik in Herodes Reich fehlten. Aber auch in anderen Klientelreichen ist das römische opus reticulatum belegt: In Cherchell in Mauretanien wurde die Bautechnik bei einem Nymphäum, das in das 1. Jh. v. Chr. datiert wird, angewandt. ${ }^{32}$ Während der Herrschaft des Ptolemaios ist sie an den königlichen Mausoleen in der Nähe derselben Stadt belegt. ${ }^{33}$ Die Retikulat-Technik wurde genutzt für die Errichtung der Stadtmauern von Samosata, der Hauptstadt des Reiches von Kommagene, ${ }^{34}$ und in Sebaste-Elaioussa an Gebäuden, die mit Archelaos, dem König von Kappadokien, in Verbindung gebracht werden. ${ }^{35}$ Die Beispiele von Retikulat-Verwendung im östlichen Mittelmeerraum und im Vorderen Orient sind umso erstaunlicher, als es keine gleichzeitigen Parallelen außerhalb der Klientelreiche gibt. $^{36}$

Ein Beispiel für die Adaption römischer Architekturkonzepte an eigene Bedürfnisse im Westen stellt der Palast von Fishbourne dar, wie jüngst $\mathrm{H}$. von Hesberg gezeigt hat. ${ }^{37}$ Die Anlage zeigt, wie Elemente der

${ }^{29}$ Allgemein: H. von Hesberg, Römische Architektur (München 2005) (mit der älteren Lit.); H.O. Lambrecht, Opus caementitium (sic!) (Düsseldorf 1984).

${ }^{30}$ S. Rocca, Herod's Iudea. A Mediterranean State in the Classical World (Stuttgart 2008), 96-127.

31 Zum Hafen allgemein: R. Hohlfelder (Hrsg.) King Herod's dream. Caesarea on the sea (New York-London 1988), 90-105; Japp 2000, a. a. O. (Anm. 13), 60-62.

32 allgemein: Japp 2000, a.a. O. (Anm. 13), 81 f.; Roller 2003, a. a. O. (Anm. 12), 121.

${ }^{33}$ P. Leveau, ,Trois tombeaux monumentaux à Cherchel', Bulletin d'Archéologie Algerienne 4 (1970), 101-148.

34 A.A. Tirpan, ,Roman masonry techniques at the capital of the Commagenian Kingdom, in: D.H. French-C.S. Lightfoot (Hrsg.), The Eastern Frontier of the Roman Empire (Oxford 1989), 519-536.

${ }^{35}$ So die Phase I der Hafenthermen: M. Spanu, ,Le terme del porto, in: E. Equini Schneider (Hrsg.), Elaiussa Sebaste II (Rom 2003), 299-301.

${ }^{36} \mathrm{M}$. Waelkens, ,The adoption of Roman building techniques in Asia minor, in: S. Macready-F.H. Thompson (Hrsg.), Roman architecture in the Greek world (London 1987), 94-105, vor allem 101.

${ }^{37}$ H. von Hesberg, ,Einheimische Bauherren und römische Architekturkonzepte im Westen des römischen Reiches', in: F. Pirson-U. Wulff-Rheidt (Hrsg.), Austausch und Inspiration: Kulturkontakt als Impuls architektonischer Innovation; Kolloquium vom 28.30.4.2006 in Berlin anlässlich des 65. Geburtstages von AdolfHoffmann (Mainz 2008), 160- 
römischen Villa übernommen, aber gleichzeitig einheimischen Vorstellungen von Repräsentation angepasst wurden, indem vor allem die Person des Besitzers, wohl Togidubnus, herausgestellt wurde.

Der Techniktransfer erfolgte wohl durch römische Handwerker, wobei unklar bleiben muss, ob es sich um Abteilungen der römischen Armee gehandelt hat oder um kaiserliche bzw. private Bauhütten. ${ }^{38}$ Treibende Kraft für die Übernahme scheint jedoch immer der König selbst gewesen zu sein, wenn man den Charakter der Bauten und deren Lokalisierung in den jeweiligen Kapitalen oder ,Palästen' berücksichtigt.

\section{b. Militärausrüstung}

Die Herrschaft der Klientelkönige beruhte in starkem Maße auf der Unterstützung durch Rom. Insofern spielte die Verbindung mit der römischen Armee als dem wichtigsten Machtinstrument eine entscheidende Rolle. Es ist allgemein bekannt, dass die reges enge Beziehungen zum Militär unterhielten. Eine besondere Qualität erhält dieser Konnex dadurch, dass er zur Repräsentation und zur Statusaffirmation oder -steigerung genutzt wurde, wobei insbesondere die Befehlsgewalt über Auxiliartruppen von den Klientelkönigen herausgestrichen wird. ${ }^{39}$ Archäologisch lässt sich dies durch den Fund römischer Militaria in Gräbern finden, die mit Klientelkönigen in Verbindung gebracht werden können: Hervorragende Bedeutung kam anscheinend den Maskenhelmen $\mathrm{zu}^{40}$ So wurde im Königsgrab von Bizye in Thrakien, der Hauptstadt des

\footnotetext{
173, vor allem 169 f.; zum Palast von Fishbourne: J. Cunliffe, Excavations at Fishbourne 1961-1969. vols. I. II (London 1971); J. Cunliffe, ,Fishbourne revisited: the site in its context', Journal of Romn Archaeology 4 (1991), 160-169; E. Black, ,Fishbourne, Chichester, and Togidubnus rex again', Journal of Roman Archaeology 21 (2008), 293-303 (mit der älteren Lit.).

${ }^{38}$ Zum Transfer von Bautechniken in der Antike: R. MacMullen, ,Roman imperial building in the provinces', Harvard Studies in Classical Philology 64 (1959), 207-235; T.F.C. Blagg, ,Roman civil and military architecture in the province of Britain: aspects of patronage, influence and craft organisation, World archaeology 12 (1980), 27-42; O. Stoll, ,Der Transfer von Technologie in der römischen Antike. Einige zusätzliche Bemerkungen zu einem Buch von Sigrid Dušek', Münsteraner Beiträge zur Antiken Handelsgeschichte 12 (1993), 93-118; K. Greene, ,Technology and innovation in context: the Roman background to mediaeval and later development', Journal of Roman Archaeology 7 (1994); O. Stoll, ,Ordinatus architectus. Römische Militärarchitekten und ihre Bedeutung für den Technologietransfer', in: L. Schumacher (Hrsg.), Religion-Wirtschaft-Technik. Althistorische Beiträge zur Entstehung neuer kultureller Strukturmuster im historischen Raum Nordafrika/Kleinasien/Syrien (St. Katharinen 1998), 203-227.

${ }^{39}$ Creighton 2006, a. a. O. (Anm. 4), 50-54.

${ }^{40}$ G. Franzius, ,Maskenhelme, in: Wolfgang Schlüter-Rainer Wiegels (Hrsg.), Rom,
} 

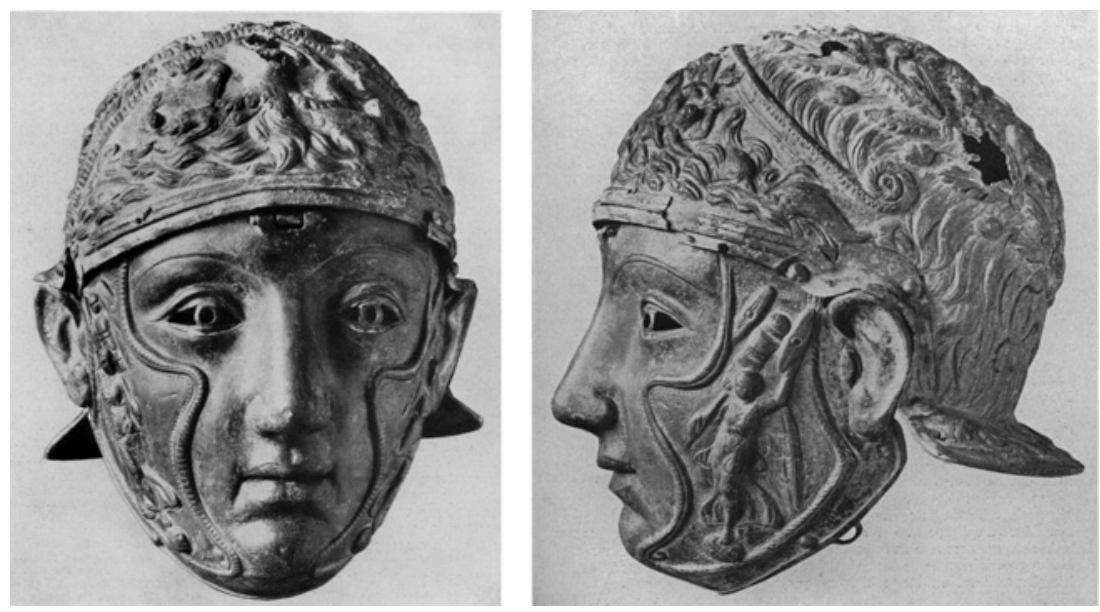

Abb. 2: Gesichtshelm aus dem Königsgrab von Bizye (Vize)

odrysischen Königreichs, neben Kettenpanzer, Schwert und zwei Lanzen ein aufwändig gestalteter Gesichtshelm (Abb. 2) gefunden. ${ }^{41}$ Ein nahezu identischer Helm ist Teil der sehr wertvollen Beigaben eines Grabes in Emesa, das aufgrund der Datierung am ehesten Iamblichus II., dem ersten Klientelkönig, zugewiesen werden kann. ${ }^{42}$

Die Bedeutung, die von einheimischen Herrschern der Verbindung mit dem römischen Heer zugemessen wurde, wird bestätigt durch die Funde qualitätvoller römischer Militärausrüstung in Bestattungen von Personen hohen gesellschaftlichen Rangs in Britannien, so in Verulamium/Saint Albans in Lexden und Folly Lane sowie in Baldock ${ }^{43}$ oder

Germanien und die Ausgrabungen von Kalkriese (Osnabrück 1999), 117-148; N. HanelU. Peltz-F. Willer, ,Untersuchungen zu römischen Reiterhelmmasken aus der Germania inferior', Bonner Jahrbücher 204 (2004), 71-91.

${ }^{41}$ A.M. Mansel, ,Grabhügelforschung im östlichen Thrakien', Archäologischer Anzeiger (1941), 119-187, vor allem 155-182; N. Başgelen, Arif Müfid Mansel's Excavations of Tumuli in Turkish Thrace (Istanbul 2009); zum Helm: G. Waurick, ,Römische Helme, in: A. Bottini (Hrsg.), Antike Helme. Sammlung Lipperheide und andere Bestände des Antikenmuseums Berlin (Mainz 1988), 360-363; Franzius 1999, a.a. O. (Anm. 40), 120; L. Hansen, Die Panzerung der Kelten. Eine diachrone und interkulturelle Untersuchung eisenzeitlicher Rüstungen (Kiel 2003), 74.

${ }^{42} \mathrm{H}$. Seyrig, ,Antiquités syriennes. Antiquités de la nécropole d'Émèse', Syria 29 (1952), 204-250; W. Ball, Rome in the East. The transformation of an empire (London 200o), 35 f.; zum Helm: Waurick 1988, a. a. O. (Anm. 41), 361-363; M. Junkelmann, Reiter wie Statuen aus Erz (Mainz 1996), 24; 120.

${ }^{43}$ Creighton 2006, a. a. O. (Anm. 4), $49 \mathrm{f}$. 
im ,Königlichen Grab` von Es Soumâa in Algerien. ${ }^{44}$ Römische Militaria können deshalb als ein weiterer Baustein für die Konstruktion der kulturell vielseitigen Identität der Klientelkönige betrachtet werden.

\section{c. Koch- und Tafelgeschirr}

Die bisherige Untersuchung konzentrierte sich ganz auf die Person des Herrschers. Um herauszufinden, ob und in welchem Umfang römische Objekte oder Kulturtechniken von breiteren Bevölkerungsschichten übernommen wurden bzw.-allgemeiner-welche Folgen die Tatsache, in einem römischen Klientelreich zu leben, für die Einwohner außer den Königen hatte, muss von anderer archäologischer Evidenz ausgegangen werden. Für die Einbeziehung dieser, auch niedrig stehender sozialer Gruppen ist in erster Linie Keramik geeignet, da sie als billiges und einfach herzustellendes Produkt weit verbreitet war und in großen Mengen zur Verfügung steht. ${ }^{45}$

In den beiden Klientelreichen Englands, im Süden und Osten der Insel, kamen in der 2. Hälfte des 2. Jhs. v. Chr. neue Keramikformen wie Teller oder Becher auf, die römische Vorbilder nachahmten. ${ }^{46}$ Einige der Gefäße waren vom Festland importiert, bei den meisten handelte es sich aber um lokal produzierte Imitationen. Da alle Gefäße der Nahrungsaufnahme dienten, spricht ihr massives Aufkommen für eine Änderung der Ess- und Trinkgebräuche in dieser Zeit, die sich durch die Übernahme römischer Tafelsitten erklären lässt. Die Analyse des Formenbestandes an unterschiedlichen Fundstätten wie Braughing, Gorhambury und dem King Harry Lane-Friedhof in Verulamium hat gezeigt, ${ }^{47}$ dass zwischen der Oberschicht und den einfacheren Gruppen der Gesellschaft deutliche Unterschiede existieren, so gibt es eine Trennung, die sich durch

${ }^{44}$ G. Waurick, ,Die Schutzwaffen im numidischen Grab von Es Soumâa', in: H.G. Horn-Ch.B. Rüger (Hrsg.), Die Numider. Reiter und Könige nördlich der Sahara (Bonn 1979), 305-332.

${ }^{45}$ Zur Rolle von römischer Keramik als Fundmaterial: J.T. Peña, Roman pottery in the archaeological record (Cambridge 2007) (mit der älteren Lit.); grundlegend zu Keramik als Indikator von kulturellen Wandlungsprozessen in Grenzgebieten: M.L. Okun, ,An example of the process of acculturation in the early Roman frontier', Oxford Journal of Archaeology 8 (1989), 41-54.

46 Zum Folgenden: H.E.M. Cool, Eating and Drinking in Roman Britain (Cambridge 2006), 155-158.

47 Cool 2006, a. a. O. (Anm. 46), 159-161; vgl. auch M. Pitts, ,Globalizing the local in Roman Britain: An anthropological approach to social change', Journal of Anthropological Archaeology 27 (2008), 497. 
das Trinken von Wein anstelle von Bier manifestiert. ${ }^{48}$ Grundsätzlich kann im vorrömischen Britannien nicht von einer Übernahme römischer Tafelsitten auf breiter gesellschaftlicher Basis gesprochen werden, eine entsprechende Übernahme von Kulturpraktiken wurde nur vom oberen Segment der Gesellschaft getragen.

Keramik lässt jedoch nicht nur auf eine-partielle-Änderung beim Verzehr, sondern auch bei der Zubereitung von Speisen schließen. Eine spezielle Gruppe von Gefäßen mit einem dicken roten Überzug im Inneren wird gewöhnlich als Pompeian Red Ware bezeichnet. ${ }^{49}$ Dabei handelt es sich meist um flache Schüsseln und Platten mit zugehörigen Deckeln. Da viele der Gefäße Rußspuren zeigen, müssen sie mit Feuer in Berührung gekommen sein und deshalb primär der Zubereitung von Speisen gedient haben. Die flachen Formen der Pompeian Red Ware sind besonders geeignet zum Backen von Kuchen oder Aufläufen, wie sie typisch für die römische Küche sind. Auch zum Backen von Brot können sie verwendet werden. Im Kochbuch des Apicius werden entsprechende Schüsseln, meist patinae genannt, für insgesamt 53 Rezepte benötigt. ${ }^{50}$ In Britannien wurde diese Keramikgattung vor allem in Militärlagern und größeren Städten des 1. Jhs. n. Chr. gefunden, aber auch vor der Eroberung in verschiedenen Orten, so in Sheepen, der Residenz des Reiches der Cattevellauni in der Nähe der späteren römischen Kolonie Colchester. ${ }^{51}$ Der Beleg von Pompeian Red Ware verweist also auf römische Kochpraktiken, vermutlich auf die Existenz entsprechend geschulter Köche, bei den Eliten des östlichen Klientelreiches. ${ }^{52}$

Pompeian Red Ware ist auch im Herrschaftsgebiet Herodes' des Großen bezeugt: In Judaea wurde diese Keramik an Stätten wie Samaria, Cae-

48 Cool 2006, a. a. O. (Anm. 46), 146-174.

49 Zur Gattung: C. Goudineau, ,Note sur la céramique à engobe interne rouge-pompéien ('Pompejanisch-roten Platten')', Mélanges d'École française à Rome 82 (1970), 159186; D.S. Peacock, ,Pompeian red ware', in: D.S. Peacock (Hrsg.), Pottery and early commerce. Characterization and trade in Roman and later ceramics (London-New YorkSan Francisco 1977), 147-162; M.C. Leotta, ,Ceramica a vernice rossa interna', in: D. Gandolfini (Hrsg.), La ceramica e i materiali di età romana. Classi, produzioni, commerci e consumi (Bordighera 2005), 115-120.

50 Apicius $3.4 .1 ; 3.3 ; 3.6 ; 3.7 ; 3.9 .3 ; 4.2 .1-37 ; 4.4 ; 4.5 .1 ; 7.4 .5 ; 7.5 .5 ; 7.13 .8 ; 7.15 .6 ; 8.6 .2-$ $3.9 ; 8.8 .1 ; 9.3 .1 ; 9.8 .3 ; 10.1 .4-5$.

51 Zur Verbreitung in Britannien: Peacock 1977, a.a.O. (Anm. 49), 158 f.; Belege in Sheepen: C.F.C. Hawkes-M.R. Hull, Camulodunum. First report on the excavations at Colchester 1930-1939 (Oxford 1947), 221 Form 17A; Cool 2006, a.a. O. (Anm. 46), 76.

52 Cool 2006, a. a. O. (Anm. 46), 166 f.; in dieselbe Richtung weist das Vorkommen von mortaria an Fundplätzen mit Elite-Charakter in Kontexten, die vor die Eroberung gesetzt werden müssen: ebenda 166 Anm. 48. 
sarea maritima, Jericho, Machairos, dem Herodion und Jerusalem gefunden, außerhalb Judaeas in Dor und Panias. ${ }^{53}$ In Jericho ist sie in der Zeit von 15 v. Chr. bis 6 n. Chr. datiert, so dass die Einfuhr aus Italien in die Herrschaftszeit von Herodes gesichert ist. ${ }^{54}$ Grundsätzlich ist Pompeian Red Ware relativ häufig in den städtischen Zentren des herodianischen Reiches und den königlichen Residenzen, aber sehr selten in ländlichen Siedlungen. Es kann deshalb angenommen werden, dass auch in Palästina wie in Britannien pompejanisch-rote Platten den Bedarf des Königs und der einheimischen Elite deckten. ${ }^{55}$ Die flachen Backschüsseln hatten insofern einen Einfluss auf die Keramikproduktion und Küche in Herodes' Reich, als während des 1 . Jhs. n. Chr. lokale Imitationen hergestellt wurden. ${ }^{56} \mathrm{Da}$ entsprechende Formen nicht im Repertoire des östlichen Mittelmeerraums vorkommen, sind sie ein Hinweis auf die Adaption römischer Kochgewohnheiten, doch war ihre Akzeptanz sehr beschränkt und ist zudem häufig nur bestimmten gesellschaftlichen Gruppen zuzuordnen, wie die Befunde aus Tell Anafa belegen. Dieser wichtige Fundort in der Nähe des Hula-Sees hat unsere Kenntnisse vom Keramikgebrauch im Reich des Herodes Philippos entschieden erweitert. ${ }^{57}$ Hier wurden deutliche Unterschiede zwischen dem Gefäßspektrum der späthellenistischen und römischen Zeit festgestellt. ${ }^{58}$ So konnten der Phase zwischen 4 v. Chr. und 50 n. Chr. offensichtlich weniger Krüge zum Servieren von Getränken und weniger Parfümfläschchen zugeordnet werden, was auf einen einfacheren Lebensstandard schließen lässt. Besonders kennzeichnend ist der Gebrauch von Backschüsseln, zum Teil auch von Pompeian Red Ware, die einen Anteil von $11 \%$ des Kochgeschirrs ausmacht, während sie für die frühere Zeit fehlen. ${ }^{59}$ Eine Übernahme römischer Kochgebräuche durch die einheimische Bevölkerung kann

53 A. Berlin, ,The plain wares', in: S.C. Herbert (Hrsg.), Tell Anafa II, i: The Hellenistic and Roman Pottery (Ann Arbor 1997), 30-32; R. Bar-Nathan, Masada VII. The Yigael Yadin excavations 1963-1965. Final reports: The pottery of Masada (Jerusalem 2006), 358360.

${ }^{54}$ R. Bar-Nathan, Hasmonean and Herodean Palaces at Jericho (Jerusalem 2002) 138140.

${ }^{55}$ R. Rosenthal-Heginbottom, ,Hellenistic and Early Roman fine ware and lamps from Area A', in: A. Geva (Hrsg.), Jewish Quarter Excavations in the Old City of Jerusalem II (Jerusalem 2003), 216f.; Bar-Nathan 2006, a. a. O. (Anm. 53), $358 \mathrm{f}$.

${ }_{56}$ Berlin 1997, a. a. O. (Anm. 53), $104 \mathrm{f}$.

57 Zur Grabung allgemein: S.C. Herbert, Tel Anafa I, i. ii: Final report on ten years of excavation at a Hellenistic and Roman settlement in Northern Israel. 10. Supplement Journal of Roman Archaeology (Ann Arbor 1994).

${ }^{58}$ Berlin 1997, a. a. O. (Anm. 53), 30-32.

59 Berlin 1997, a. a. O. (Anm. 53), 104-109. 
jedoch deshalb nicht angenommen werden, zumal auch weitere typische Gefäßformen wie mortaria fehlen. ${ }^{60}$ Am wahrscheinlichsten ist, dass Soldaten in Tell Anafa stationiert waren, deren Essgewohnheiten jedoch nicht allgemein übernommen wurden. Trotzdem zeigt die Keramik in Tell Anafa, dass auch einfache Siedlungen in Klientelreichen Kontaktzonen waren, wobei freilich nicht immer reger Kulturaustausch herrschen musste, sondern es auch zu einem Nebeneinander zweier Lebensstile kommen konnte.

\section{KlientelkönigtüMer als Thema von Frontier StUdies}

Die genauere Betrachtung vor allem der materiellen Kultur in den Klientelkönigreichen hat gezeigt, dass sie als Kontaktzonen Regionen erhöhten kulturellen Austauschs waren. ${ }^{61}$ Da diese Gebiete nicht linear als border, sondern nur gebietsweise als frontiers zu verstehen sind, kann die Beschäftigung mit ihnen dazu verhelfen, einige Versäumnisse und Probleme von frontier studies im Allgemeinen zu benennen und sich ihrer bewusst zu werden, so vor allem die einheimische Bevölkerung vom König bis zu den einfachen Untertanen zu ignorieren und zu marginalisieren. ${ }^{62}$ Ganz in diesem Sinn sollten die Bewohner der Grenzregionen des Imperium Romanum selbst als handlungsmächtige Agenten in Prozessen kulturellen Wandels gesehen werden. ${ }^{63}$ Zwei Probleme sind insbesondere zu nennen:

60 Berlin 1997, a.a. O. (Anm. 53), 123-126; A. Berlin, ,Italian cooking vessels and cuisine from Tel Anafa', Israel Exploration Journal 43 (1993), 35-44.

61 ,Kontakt' und ,Kontaktzonen' sind Kernbegriffe der altertumswissenschaftlichen Forschung: K.G. Lightfoot, ,Culture contact studies', American Antiquity 60 (1995), 199217; J. Cusick, Studies in culture contact: interaction, culture change and archaeology (Carbondale 1998); R. Rolle-K. Schmidt (Hrsg.), Archäologische Studien in Kontaktzonen der antiken Welt (Göttingen 1998); C. Gosden, Archaeology and colonialism. Culture contact from $5000 \mathrm{BC}$ to the present (Cambridge 2004).

62 Die Unterscheidung von ,border ‘ und ,frontier' lässt nicht durch das deutsche Wort ,Grenze‘ wiederholen; vgl. Braund 1984, a.a. O. (Anm. 3), 95; methodisch grundlegend: P. Southern, ,Comparative frontier studies', in: E. Scott (Hrsg.), Theoretical Roman Archaeology: First Conference Proceedings (Avebury 1994), 147-154; K.G. LightfootA. Martinez, ,Frontiers and boundaries in archaeological perspective', Annual Reviews of Anthropology (1995), 471-492; C.M. Wells, ,Profuit invitis te dominante capi: social and economic considerations on the Roman frontiers', Journal of Roman archaeology 9 (1996), 436-446; A. Gardner, ,Fluid frontiers: cultural interaction on the edge of empire, Stanford Journal of Archaeology 5 (2007), 43-6o.

63 Zum Konzept der agency in der Archäologie: M.-A. Dobres-J.E. Robb (Hrsg.), Agency in archaeology (London-New York 200o); J.L. Dornan, ,Agency and archaeology: 
1.) Historische Untersuchungen der regna behandelten meist das Gebiet in seiner Gesamtheit, galten also der Makroregion. Archäologische Analysen beschränken sich meist auf eine oder einige wenige Ausgrabungen, sind also auf Mikroregionen bezogen. Es ist notwendig, beide Herangehensweisen miteinander zu verbinden, da nur auf diese integrierte Weise sowohl die spezifischen Formen als auch die weiteren Effekte des Kulturkontakts nachgezeichnet werden können.

2.) Die Studien zu Klientelkönigen und ihren Herrschaftsgebieten unterstützen notwendigerweise ein ,top down'-Modell des Kulturwandels, das eher Entwicklungen am Königshof in den Fokus stellt als solche in anderen gesellschaftlichen Bereichen. Es liegt natürlich im Interesse der Eliten an der Peripherie, in erster Linie des Königs selbst, die engen Beziehungen zu Rom als der wichtigsten Legitimation seiner Herrschaft herauszustellen. Es ist jedoch unbedingt zu fragen, ob andere soziale Gruppen der Bevölkerung ihre eigenen speziellen Identitäten in diesem Grenzbereich kreierten, insbesondere inwieweit die starke Bezugnahme auf Rom, die der König praktizierte, übernommen wurde. ${ }^{64}$ Trotz Schwierigkeiten bei der Quellenlage kann durch Analyse der archäologischen Evidenz, insbesondere von Massenprodukten wie Keramik, gezeigt werden, dass die Beeinflussung seiner eigenen Untertanen durch Praktiken und

past, present, and future directions', Journal of Archaeological Method and Theory 9 (2002), 303-329; A. Gardner (Hrsg.), Agency Uncovered: archaeological perspectives on social agency, power, and being human (London 2004).

64 ,Identität' ist in den letzten Jahren zum zentralen Paradigma innerhalb der römischen Archäologie geworden und hat-berechtigterweise-den zu pauschalen und historisch belasteten Terminus Romanisierung ersetzt: A. Gardner, ,Social identity and the duality of structure in late Roman Britain, Journal of social archaeology 2 (2002), 323351; D. Mattingly, ,Being Roman: expressing identity in a provincial setting', Journal of Roman Archaeology 17 (2004), 5-25; A. Schmidt-Colinet (Hrsg.), Lokale Identitäten in Randgebieten des römischen Reiches. Akten des Internationalen Symposiums in Wiener Neustadt, 24.-26. April 2003 (Wien 2004); A. Gardner, An Archaeology of Identity: soldiers and society in late Roman Britain (Walnut Creek 2007); R. Roth-J. Keller (Hrsg.) Roman by Integration: dimensions of group identity in material culture and text. 66. Supplement Journal of Roman Archaeology (Providence 2007); S. Hinds-T. Schmitz, ,Constructing identities in the Roman Empire: three studies', Millenium 4 (2007), 1-12; M. Pitts, ,The emperor's new clothes? The utility of identity in Roman archaeology', American Journal of Archaeology 111 (2007), 693-713; M. Sommer, ,Bauen an der Grenze. Überlegungen zur Monumentalisierung kultureller Identitäten', in: F. Pirson-U. Wulff-Rheidt (Hrsg.), Austausch und Inspiration: Kulturkontakt als Impuls architektonischer Innovation; Kolloquium vom 28.-30.4.2006 in Berlin anlässlich des 65. Geburtstages von Adolf Hoffmann (Mainz 2008), 202-213; L. Revell, Roman imperialism and local identities (Cambridge 2009) (jeweils mit weiterer Lit.). 
Formen der römischen Kultur, die vom König demonstrativ aufgegriffen wurden, gering ist. Es kommt also in Klientelkönigreichen-im Unterschied zu vielen Provinzen des Römischen Reiches-nur sehr begrenzt zu einem ,trickle down'-Effekt. ${ }^{65}$ Die teilweise unmittelbar an die Person des Königs gebundene Beziehung zwischen Klientelreich und Rom bzw. Italien kann archäologisch am besten anhand von Lebenmittellieferungen exemplifiziert werden: In Masada fand man Amphoren, die laut Aufschrift direkt für Herodes bestimmt waren und Wein und Äpfel aus Italien sowie garum aus Spanien enthielten. ${ }^{66}$

Im Unterschied zum Zentrum-Peripherie-Modell, wo das Zentrum die entscheidende Rolle bei der Veränderung kulturellen Wandels innehatte, spielte bei Klientelkönigreichen die Peripherie den aktiven Part. ${ }^{67}$ Zwar wurden im Rahmen der Erziehung der Prinzen als obsides in Rom periphere Elemente ins Zentrum gebracht, doch wurden sie nach ihrer Erziehung an den äußeren Rand gesandt. ${ }^{68}$ Somit waren die Könige Agenten des kulturellen Wandels, freilich unterschied sich deren kulturelle Identität nachdrücklich von der seiner ,Landsleute, die deutlich vielgliedriger war und eine dezidiert römische Facette aufwies. So sind auch Gemeinsamkeiten in der Repräsentation der reges zu erklären. ${ }^{69}$

${ }^{65}$ Grundlegend: M. Millett, The romanization of Britain (Cambridge 1990).

${ }^{66}$ So z. B. Regi Herodi Iudaico: H.M. Cotton-J. Geiger, Masada II: The Yigael Yadin excavations 1963-1965. Final report: The Latin and Greek documents (Jerusalem 1989), passim; Bar-Nathan 2006, a. a. O. (Anm. 53), 312-314.

${ }^{67}$ T. Champion (Hrsg.), Centre and Periphery. Comparative Studies in Archaeology (Cambridge 1989); M. Rowlands-K. Kristiansen (Hrsg.), Centre and Periphery in the Ancient World (London-New York 1998); G. Schörner, ,Das Zentrum-PeripherieModell in der Romanisierungsforschung,, in: G. Schörner (Hrsg.), RomanisierungRomanisation. Theoretische Modelle und praktische Fallbeispiele (Oxford 2005), 95-99 (mit weiterer Lit.); zum Zentrum-Peripherie-Modell in frontier studies: P.S. Wells, ,Production within and beyond imperial boundaries: goods, exchange, and power in Roman Empire, in: N. Kardulias (Hrsg.), World-Systems theory in practice: leadership, production, and exchange (Lanham 1996), 85-101.

68 Allgemein: J. Gagé, Res gestae divi Augusti (3. Auflage, Paris 1977), 142 f.; Braund 1984, a. a. O. (Anm. 3), 15-17; ein besonders gut erforschtes Beispiel: M. Hadas-Lebel, ,Léducation des princes hérodiens à Rome et l'évolution du clientélisme romain', in: M. Mor u. a. (Hrsg.), Jews and gentiles in the Holy Land in the days of the Second Temple, the Mishna and the Talmud (Jerusalem 2003), 44-62; obsides aus dem Westen: Creighton 2000, a. a. O. (Anm. 4), 91-93.

${ }^{69}$ So werden identische Münztypen, die auf Prägungen Roms zurückgehen, gleichermaßen von Tincomarus, Verica und Epaticcus in Britannien sowie Iuba I, Iuba II und Ptolemaios in Mauretanien verwendet, außerdem von Königen Noricums und dem treverischen Fürsten Arda: Creighton 2000, a. a. O. (Anm. 4), 118-122 Abb. 4.12. Zum römischen Vorbild: RRC 361. 
Dass dabei deren Ausdrucksformen aus dem Rahmen des regional Typischen fallen können, verdeutlicht die Münzprägung. So unterscheiden sich die Münzen der Reiche im Osten deutlich von denen der Poleis in ihrer unmittelbaren Nachbarschaft. ${ }^{70}$ Jene ahmen stadtrömische Prägungen nach, ganz ähnlich wie auch in den Klientelkönigreichen im Westen, während diese einen viel stärkeren Lokalbezug aufweisen und vor allem lokale Mythen und Spiele thematisieren. Teilweise wird, wie im Falle von Agrippa, sogar Latein für die Titulatur benutzt. ${ }^{71}$

Der Grund für diesen ungemein engen Anschluss an römische Formen ist evident: Die reges mussten ihre festen Beziehungen mit Rom als ihren raison dêtre herausstreichen und immer wieder augenfällig machen.

Das grundsätzliche Prinzip, nämlich dass materielle Kultur römischer Prägung innerhalb des Imperiums in lokalen Gesellschaften als Statussymbol genutzt werden konnte, gilt auch für die Klientelreiche. ${ }^{72}$ Es gibt jedoch ein signifikantes Charakteristikum: Die Adoption römischer Kultur bezieht sich meist auf die Könige und ist auch hauptsächlich von ihnen veranlasst. Im Unterschied $\mathrm{zu}$ den Provinzen war der Prozess des kulturellen Wandels in den Klientelreichen deshalb ungleichmäßiger und kurzlebiger: ungleichmäßig insofern, als manche Bereiche der indigenen Kultur viel stärker durch römische Modelle beeinflusst sind als andere, häufig auch in einer Art und Weise, wie sie nicht mit den regulären Provinzen des Römischen Reiches vergleichbar ist, wie die Bauten in opus reticulatum oder die Münzen mit lateinischen Legenden beweisen. ${ }^{73}$ Gleiches gilt für die Porträts der mauretanischen Könige im besten römischen Stil $^{74}$ oder die Wanddekoration zweiten Stils in Masada. ${ }^{75}$

${ }^{70}$ A. Burnett, ,The Roman West and the Roman East', in: Ch. Howgego-V. Heuchert-A. Burnett (Hrsg.), Coinage and identity in the Roman provinces (Oxford 2005), $171-180$.

${ }^{71}$ Burnett 2005, a. a. O. (Anm. 70), 178.

72 Grundlegend: P.S. Wells, ,Identity and material culture in the Later Prehistory of Central Europe;, Journal of Archaeological Research 6 (1998), 239-298; vgl. auch P.W.M. Freeman, ,'Romanisation' and material culture', Journal of Roman archaeology 6 (1993), 438-445.

${ }_{73}$ Hier Anm. 29; Burnett 2005, a. a. O. (Anm. 70), 178.

${ }^{74}$ K. Fittschen, ,Bildnisse numidischer Könige, in: H.G. Horn-Ch.B. Rüger (Hrsg.), Die Numider. Reiter und Könige nördlich der Sahara (Bonn 1979), 209-225.

${ }^{75}$ G. Foerster, Masada V. The Yigael Yadin excavations 1963-1965. Final reports: Art and architecture (Jerusalem 1995), 22-60; K. Fittschen, ,Wall decorations in Herod's kingdom: their relationship with wall decorations in Greece and Italy', in: K. Fittschen- 
Kurzlebiger vor allem deshalb, weil die Annexion der Klientelreiche einen Wandel der materiellen Kultur nach sich zieht, die einheimischer wird beziehungsweise in erster Linie stärkeren lokalen Charakter annimmt. Beispiele hierfür sind das Verschwinden von Pompeian Red ware außerhalb von Städten und Militäranlagen im römischen Britannien oder das Keramikspektrum in Masada nach der Ära Herodes des Großen. ${ }^{76}$ Deshalb scheint die Kultur in den Klientelreichen noch mehr fragmentiert zu sein und noch mehr Ergebnis von ,bricolage zu sein, als dies N. Terrenato für die regulären Provinzen angenommen hat. ${ }^{77}$ Freilich ist es sehr fraglich, ob die Klientelkönige überhaupt die Schaffung einer möglichst homogenen Kultur römischer Prägung in ihren Reichen anstrebten oder ob sie durch ihre Bindung an Rom, die vom Rest der Bevölkerung nicht nachvollzogen wurde, ihre kulturelle Isolierung in Kauf nahmen.

Jedenfalls sind die Klientelreiche sehr gute Beispiele dafür, dass wir grundsätzlich Grenzen eher als Zonen kulturellen Kontakts zu sehen haben und nicht als scharfe Trennlinien. Die Bewohner dieser Regionen können deshalb eine Vielfalt unterschiedlicher kultureller Identitäten ausbilden, wobei in den Klientelkönigreichen die Möglichkeiten zwischen enger Anlehnung an Rom und Festhalten an der traditionellen Kultur besonders groß sind. Grundsätzlich erfordert es diese aktive Rolle, die die Bewohner in den regna einnehmen, unsere fest gefügten Vorstellungen vom römischen Imperialismus beziehungsweise Kolonialismus und vom Verhältnis von Zentrum und Peripherie in Frage zu stellen und einer kritischen Prüfung zu unterziehen. ${ }^{78}$

Jena, Januar 2010

G. Foerster (Hrsg.), Judaea and the Greco-Roman World in the Time of Herod in the Light of Archaeological Evidence (Göttingen 1996), 139-161; vgl. auch S. Rozenberg, ,The wall paintings of the Herodian Palace at Jericho, in: Fittschen-Foerster 1996, a.a. O., 121138.

76 Hier Anm. 51 und 55.

77 N. Terrenato, The Romanization of Italy: global acculturation or cultural bricolage?, in: C. Forcey-J. Hawthorne-R. Witcher (Hrsg.), Theoretical Roman Archaeology Conference 1997 (Oxford 1998), 20-27.

${ }^{78} \mathrm{Zu}$ Kolonialismus und Imperialismus aus archäologischer Sicht: B. Bartel, ,Colonialism and cultural responses: problems related to Roman provincial analysis, World Archaeology 12 (1980), 11-26; R. Hingley, ,Roman Britain: the structure of Roman imperialism and the consequences of imperialism in the development of a peripheral province, in: D. Miles (Hrsg.), The Romano-British countryside (Oxford 1982), 17-52; 


\section{Abbildungsnachweis}

Abb. 1a S. Reinach, Répertoire des reliefs grecs et romains I (Paris 1909) 419. Abb. 1b S. Reinach, Répertoire des reliefs grecs et romains I (Paris 1909) 420. Abb. 2a A.M. Mansel, ,Grabhügelforschung im östlichen Thrakien', Archäologischer Anzeiger 1941, Abb. 31.

Abb. 2b A.M. Mansel, ,Grabhügelforschung im östlichen Thrakien', Archäologischer Anzeiger 1941, Abb. 33.

J. Webster-N. Cooper (Hrsg.), Roman Imperialism: post-colonial perspectives (Leicester 1996); D. Mattingly, Dialogues in Roman Imperialism. Power, discourse and discrepant experience in the Roman Empire. 23. Supplement Journal of Roman Archaeology (Portsmouth 1997); Gosden 2004, a. a. O. (Anm. 61); G. Schörner, Imperialismus, Kolonialismus und Postkolonialismus in der Romanisierungsforschung, in: Schörner 2005, a. a. O. (Anm. 67), 25-34; M. Given, The archaeology of the colonized (London 2004); Revell 2009, a. a. O. (Anm. 64); zu Zentrum und Peripherie: hier Anm. 67. 